\title{
CAPÍTULO 19: CARACTERIZAÇÃO DOS PARÂMETROS MICROBIOLÓGICOS DE QUEIJO COALHO ARTESANAL PRODUZIDO NO POLO LEITEIRO DE SERGIPE
}

\author{
CAPÍTULO 19: CARACTERIZACIÓN DE PARÁMETROS MICROBIOLÓGICOS Y \\ FÍSICO-QUÍMICOS DEL QUESO DE CUAJO ARTESANAL ELABORADO
}

\section{CHAPTER 19: CHARACTERIZATION OF MICROBIOLOGICAL AND PHYSICAL- CHEMICAL PARAMETERS OF ARTISANAL RENNET CHEESE PRODUCED}

\author{
Alane Kathielle Silva Medeiros ${ }^{1}$; Maria José Soares do Santos²; João Paulo Natalino de $\mathrm{Sa}^{3}$.
}

DOI: $\underline{\text { https://doi.org/10.31692/978-65-88970-18-8.257-276 }}$

\begin{abstract}
RESUMO
De acordo com a Lei Estadual No 8.523 DE 29 de abril de 2019, é considerado, dentre outros atributos, como queijo de coalho artesanal, o produzido em Sergipe, a partir do leite fresco e cru de bovinos e bubalinos, que apresente consistência firme, cor e sabor próprios, massa uniforme, isenta de corantes e conservantes, com ou sem olhaduras mecânicas (SERGIPE, 2019). Em Sergipe, a cidade de Nossa Senhora da Gloria, se destaca como o maior polo leiteiro do estado. Segundo Cavalcante (2005) estimase que, cerca de $40 \%$ a $50 \%$ da produção de leite neste município, seja destinado à produção de queijo coalho artesanal. Neste contexto, este trabalho teve como objetivo, caracterizar os parâmetros microbiológicos de queijo coalho artesanal produzido em Nossa Senhora da Glória (SE), maior polo leiteiro de Sergipe. A metodologia aplicada foi desenvolvida a partir de seis (6) amostras de queijo coalho artesanal de diferentes fabricantes da região, onde foram efetuadas análises microbiológicas para a quantificação de Salmonella sp, Staphylococcus aureus coagulase positiva, coliformes termotolerantes e Listeria monocytogenes. Importante ressaltar, que foram incluídos nesta pesquisa somente amostras de queijo coalho artesanal sem registro de inspeção (federal, estadual e/ou municipal) e que não apresentaram alterações em suas características sensoriais e macroscópicas. Após a quantificação microbiana os resultados obtidos foram comparados com os parâmetros estabelecidos pela I.N $\mathrm{N}^{\circ} 60$, de 2019 (MAPA). Os resultados obtidos das análises demostraram que todas as amostras de queijo coalho artesanal analisadas (100\%), apresentaram valores superiores de $S$. aureus coagulase positiva e de coliformes termotolerantes com o estabelecido pela legislação vigente. Com relação a Salmonella sp foi possível constatar que $33,33 \%$ das amostras analisadas estavam fora do padrão exigido pela Instrução Normativa $\mathrm{N}^{\circ} 60 / 2019$, contudo, foi verificado a ausência de L. monocytogenes para todas as amostras de queijo coalho artesanal. Com base nesses resultados, conclui-se que os queijos coalho artesanal pesquisados estão em não conformidade com a legislação vigente, tornando-os inadequados para o consumo humano, uma vez que a sua ingestão pode ocasionar infecção e/ou intoxicação alimentar devido à presença em níveis elevados de microrganismos patogênicos (S. aures, Salmonella sp., e coliformes termotolerantes). Diante disso, sugere-se que as Boas Práticas de Fabricação durante toda a etapa de produção de queijo coalho artesanal, necessita ser aperfeiçoada, para que os parâmetros microbiológicos possam ser melhorados e atender, de forma segura, as novas necessidades e anseios do mercado, do consumidor, do produtor e dos órgãos legislatórios vigentes no Brasil.
\end{abstract}

Palavras-Chave: Qualidade microbiológica, Nossa Senhora da Glória, Legislação.

\section{RESUMEN}

De acuerdo con la Ley del Estado $\mathrm{N}^{\circ} 8.523$ de 29 de abril de 2019, se considera, entre otros atributos, como queso de cuajo artesanal, el producido en Sergipe, a partir de leche fresca y cruda de bovino y búfala, que presenta consistencia firme, color y sabor propios masa uniforme, libre de colorantes y conservantes, con o sin miradas mecánicas (SERGIPE, 2019). En Sergipe, la ciudad de Nossa Senhora

\footnotetext{
${ }^{1}$ Agroindústria, Universidade Federal de Sergipe, alanesilva@academico.ufs.br

${ }^{2}$ Agroindústria, Universidade Federal de Sergipe, mariasoares@academico.ufs.br

${ }^{3}$ Doutor, Universidade Federal de Sergipe, ipsadesa@academico.ufs.br
} 
da Gloria, se destaca como el centro lácteo más grande del estado. Según Cavalcante (2005) se estima que alrededor del $40 \%$ al $50 \%$ de la producción de leche en este municipio se destina a la producción de queso cuajo artesanal. En este contexto, este trabajo tuvo como objetivo caracterizar los parámetros microbiológicos del queso de cuajo artesanal producido en Nossa Senhora da Glória (SE), el mayor polo lácteo de Sergipe. La metodología aplicada se desarrolló a partir de seis (6) muestras de queso de cuajo artesanal de diferentes fabricantes de la región, donde se realizaron análisis microbiológicos para cuantificar Salmonella sp, coagulasa positiva Staphylococcus aureus, coliformes termotolerantes y Listeria monocytogenes. Es importante resaltar que solo se incluyeron en esta investigación muestras de queso de cuajo artesanal sin registro de inspección (federal, estatal y / o municipal) y que no presentaron cambios en sus características sensoriales y macroscópicas. Tras la cuantificación microbiana, los resultados obtenidos se compararon con los parámetros establecidos por el I.N N ${ }^{\circ} 60$, de 2019 (MAPA). Los resultados obtenidos de los análisis mostraron que todas las muestras de queso de cuajo artesanal analizadas $(100 \%)$, presentaron valores superiores de coagulasa positiva a coagulasa positiva S. aureus y de coliformes termotolerantes con lo establecido por la legislación vigente. Con respecto a Salmonella $s p$, se encontró que el 33,33\% de las muestras analizadas estaban fuera del estándar requerido por la Instrucción Normativa No. 60/2019, sin embargo, se verificó la ausencia de L. monocytogenes para todas las muestras de queso cuajo artesanal. En base a estos resultados, se concluye que los quesos de cuajo artesanal investigados no cumplen con la legislación vigente, por lo que no son aptos para el consumo humano, ya que su ingestión puede provocar infección y / o intoxicación alimentaria por la presencia en niveles elevados de microorganismos patógenos. (S. aures coagulasa positiva, Salmonella sp. y coliformes termotolerantes). Por tanto, se sugiere mejorar las Buenas Prácticas de Fabricación durante toda la etapa de elaboración del queso de cuajo artesanal, para que se puedan mejorar los parámetros microbiológicos y atender de forma segura las nuevas necesidades y deseos del mercado, del consumidor, del productor y los cuerpos legislativos vigentes en Brasil.

Palabras Clave: Calidad microbiológica, Nossa Senhora da Glória, Legislación.

\section{ABSTRACT}

According to State Law No. 8,523 of April 29, 2019, it is considered, among other attributes, as artisanal rennet cheese, that produced in Sergipe, from fresh and raw bovine and buffalo milk, which presents firm consistency, own color and flavor, uniform mass, free from dyes and preservatives, with or without mechanical glances (SERGIPE, 2019). In Sergipe, the city of Nossa Senhora da Gloria, stands out as the largest dairy hub in the state. According to Cavalcante (2005) it is estimated that about $40 \%$ to $50 \%$ of milk production in this municipality is destined to the production of artisanal rennet cheese. In this context, this work aimed to characterize the microbiological parameters of artisanal rennet cheese produced in Nossa Senhora da Glória (SE), the largest dairy hub in Sergipe. The applied methodology was developed from six (6) samples of artisanal rennet cheese from different manufacturers in the region, where microbiological analyzes were performed to quantify Salmonella $s p$, positive coagulase Staphylococcus aureus, thermotolerant coliforms and Listeria monocytogenes. It is important to emphasize that only samples of artisanal rennet cheese without inspection record (federal, state and / or municipal) were included in this research and that did not present changes in their sensory and macroscopic characteristics. After microbial quantification, the results obtained were compared with the parameters established by I.N N ${ }^{\circ} 60$, of 2019 (MAPA). The results obtained from the analyzes showed that all the samples of artisanal rennet cheese analyzed (100\%), presented higher values of positive coagulase $S$. aureus and of thermotolerant coliforms with the established by the current legislation. Regarding Salmonella sp, it was found that $33.33 \%$ of the analyzed samples were outside the standard required by Normative Instruction No. 60/2019, however, the absence of L. monocytogenes was verified for all samples of artisanal rennet cheese. Based on these results, it is concluded that the researched artisanal rennet cheeses are not in compliance with current legislation, making them unsuitable for human consumption, since their ingestion can cause infection and / or food poisoning due to the presence in high levels of pathogenic microorganisms (positive coagulase S. aures, Salmonella sp., and thermotolerant coliforms). Therefore, it is suggested that the Good Manufacturing Practices during the entire production stage of artisanal rennet cheese, needs to be improved, so that the microbiological parameters can be improved and safely meet the new needs and desires of the market, of the consumer, the producer and the legislative bodies in force in Brazil.

Keywords: Microbiological quality, Nossa Senhora da Glória, Legislation. 
De acordo com a Lei Estadual No 8.523 DE 29 de abril de 2019, é considerado, dentre outros atributos, como queijo de coalho artesanal, o produzido em Sergipe, a partir do leite fresco e cru de bovinos e bubalinos, que apresente consistência firme, cor e sabor próprios, massa uniforme, isenta de corantes e conservantes, com ou sem olhaduras mecânicas (SERGIPE, 2019).

Em Sergipe, a cidade de Nossa Senhora da Gloria se destaca como o maior polo leiteiro do estado. Segundo Cavalcante (2005) estima-se que cerca de $40 \%$ a 50\% da produção de leite neste município seja destinado à produção de queijo coalho artesanal.

Embora o processo de produção de queijo coalho artesanal seja considerado tecnicamente como um processo simples, a sua produção nos últimos anos, vem tentando periodicamente ser aperfeiçoada com relação aos cuidados com a qualidade do produto, visando assim, atender aos anseios e segurança do consumidor, além dos critérios preconizados pela legislação vigente, como por exemplo, a obtenção do Selo Arte (MENEZES, 2017; TEIXEIRA, 2013).

Alguns estudos na literatura sobre a qualidade microbiológica de queijo coalho artesanal relataram a ocorrência de padrões microbiológicos (Salmonella spp., Escherichia coli e Staphylococcus aureus) que excederam aos limites estabelecidos pela legislação vigente industrial (KASK, et al, 2003; CRUZ, 2009; MENESES et al., 2010). Entretanto é possível constar na literatura cientifica que estes mesmos parâmetros microbiológicos para o queijo coalho artesanal são relativamente pequenos e/ou desatualizados para o estado de Sergipe, em comparação aos demais estados do Brasil.

Além destes fatores supracitados, novas regulamentações para os parâmetros microbiológicos foram implementadas no Brasil, como por exemplo, a Resolução - RDC N ${ }^{\circ}$ 331, DE 23 de dezembro de 2019 e a Instrução Normativa IN n 60 de 2019, ambas regulamentas pela Agencia Nacional de Vigilância Sanitária (ANVISA).

Neste contexto, o diagnóstico com relação aos parâmetros microbiológicos de queijo coalha artesanal produzidos no maior polo leiteiro do estado, pode contribuir para a obtenção de um derivado lácteo que possa atender as novas necessidades e anseios do mercado, do consumidor, do produtor e dos órgãos legislatórios vigentes no Brasil. 


\section{Queijo coalho}

O queijo coalho artesenal é aquele elaborado com leite integral, sendo fresco, cru ou com tratamento térmico simples, em pequena escala de produção, que se utiliza de micro ou pequena estrutura física, elaborado por métodos tradicionais, com vinculação e valorização territorial, regional ou cultural que lhe conferem identidade, com vinculação ao território de origem, conforme Regulamento Técnico de Identidade e Qualidade (RTIQ) (BRASIL,2019).

Os requisitos sensoriais do queijo coalho artesanal, incluem uma consistência semidura e/ou elástica; textura compacta e macia, apresentando uma coloração branco amarelado uniforme; sabor brando e ligeiramente ácido, podendo ser salgado; odor ligeiramente ácido, lembrando massa coagulada; crosta: fina, sem trinca, não sendo usual a formação de casca bem definida; as olhaduras quando presentes, devem ser pequenas, e o formato e peso são variáveis (BRASIL, 2001).

O queijo coalho artesanal pode ser fabricado com leite cru, desde que sejam maturados por um período inferior a 60 (sessenta) dias, e quando estudos técnico-científicos comprovarem que a redução do período de maturação não compromete a qualidade e a inocuidade do produto, ficando restrita a queijarias situadas em região de indicação geográfica certificada ou tradicionalmente reconhecida e em propriedade certificada oficialmente como livre de tuberculose e brucelose (BRASIL, 2011).

\section{Produção de queijo coalho em Nossa Senhora da Glória-SE}

No Brasil, a região nordestina é a que mais se destaca pela alta quantidade de queijos coalho artesanais produzidos, embora a produção destes queijos tenha se estendido para outras regiões do Brasil. Dentre as regiões do nordeste brasileiro, o estado de Sergipe, mais precisamente na região do semiárido, tem-se a maior bacia leiteira do estado, tendo como principal produtor o município de Nossa Senhora da Glória (JESUS et al., 2018).

A cidade de Nossa Senhora da Glória, considerada a capital do leite do Alto Sertão Sergipano, possui uma área de 756,49 km², ficando a $126 \mathrm{~km}$ de distância da cidade de Aracaju, a capital do estado. A produção leiteira deste município chegou a um total de 34.276 (x 1000) litros em 2017 (BRASIL, 2019; IBGE, 2017). A tradicional produção de queijos artesanais no Alto Sertão de Sergipe ganhou a abordagem política do arranjo produtivo local, em 2007, como 

recursos técnicos, financeiros e de infraestrutura do estado (VIEIRA; SOARES, 2019).

No município de Nossa Senhora da Glória cerca de 50 a $70 \%$ da produção de queijos, dentre estes, o queijo coalho artesanal, são absorvidos pelas pequenas indústrias, chamadas popularmente de fabriquetas. $\mathrm{O}$ consumo desta produção ocorre em parte, pela população do próprio município e parcialmente pela capital do estado (Aracaju) e por outros municípios dos estados nordestinos (MENEZES, 2004).

No entanto, embora a cidade de Nossa Senhora da Glória, apresente importante destaque na produção de queijo coalho artesanal no estado de Sergipe, é possível encontrar na literatura, trabalhos que mencionam as deficiências no arranjo produtivo, notadamente no que se refere aos padrões de higiene na coleta, no manuseio, nas condições técnicas de fabricação, no acondicionamento, na conservação desses produtos e/ ou da matéria-prima utilizada para a fabricação do queijo coalho artesanal, fatores estes que podem favorecer para a contaminação microbiana (MELO, 2020; SANTOS, 2020).

O queijo coalho artesanal contaminado por microrganismos contaminantes pode causar modificações indesejáveis no aspecto sensorial, riscos para a saúde do consumidor através de infecções e intoxicações alimentar, além de alterações nos parâmetros físico-químico (ECKERT; WEBBER, 2016).

Todos esses fatores limitam a comercialização do queijo coalho artesanal, afetando diretamente e/ou indiretamente à economia da região do Alto Sertão Sergipano. Além disso, é possível observar que, em geral, não há um controle sobre as condições de sanidade do gado leiteiro e se este queijo atende às recomendações técnicas contidas em normas e regulamentos sanitários em vigor (SANTOS et al., 2008; FRAGA et al., 2019).

\section{Características microbiológicas do queijo coalho}

Dentre os produtos derivados do leite, o queijo é considerado um alimento propicio para a multiplicação de patógenos de origem alimentar. Os queijos frescos artesanais por serem, na maioria das vezes, elaborados a partir de leite cru e não sofrerem processo de maturação, tendem a ser os mais propensos para a propagação desses microrganismos, levando a perdas econômicas para indústria e para o produtor, além de oferecer perigo à saúde pública, pelo risco de causar intoxicação e / ou infecção alimentar (FEITOSA et al., 2003; FREITAS, 2015).

Em geral, dentre os microrganismos encontrados no queijo de coalho, em função da ausência e/ou ineficiente implementação de BPF, destaca-se coliformes totais e termotolerantes, 
Salmonella sp., Staphylococcus aureus e Listeria monocytogenes (SANTANA et al., 2008; BONFIM, et al., 2020).

Coliformes totais são bactérias que possuem formato de bastonetes gram-negativos, não esporulado, aeróbios ou anaeróbios facultativos, e conseguem fazer a fermentação da lactose produzindo gás, em um período de 24 a $48 \mathrm{~h}$ em temperatura de $35^{\circ} \mathrm{C}$. Já o grupo dos coliformes termotolerantes tem o mesmo conceito dos coliformes totais, mas só fazem parte desse grupo, bactérias que fazem a fermentação da lactose produzindo gás, em um tempo de $24 \mathrm{~h}$ com temperaturas que variam entre 44,5 e 45,5 ${ }^{\circ} \mathrm{C}$. Esses grupos são compostos, principalmente, por bactérias da família Enterobacteriaceae, como bactérias dos gêneros Escherichia spp., Enterobacter spp., Citrobacter spp. e Klebsiella spp (CARDOSO, 2001; CUNHA, 2006; SALES et al., 2015).

Escherichia coli (coliformes termotolerante) é uma bactéria Gram negativa, que também pertence à família das enterobacteracea. Esta espécie é bastante pesquisada devido ao risco que ela proporciona a saúde pública por desenvolver doenças entéricas como diarreias, vômitos, febre e dores abdominais. É uma bactéria que pode ser encontrada no trato intestinal de animais, inclusive em seres humanos (DRUMOND, 2018; SILVA et al., 2019; SVS, 2018; BARBOZA, 2018).

Algumas cepas de E. coli, conseguem se multiplicarem em valores de pH baixos, porém apresentam incapacidade de crescerem em temperaturas igual ou superior a $44,5{ }^{\circ} \mathrm{C}$. Uns dos alimentos geralmente associados a contaminação por essa bactéria são carne de hambúrguer mal cozida e queijos que não utilizaram a pasteurização do leite na produção (ECHEVERRY, 2004; REID, 2001; FROZI, 2017).

Na família enterobacteracea um dos gêneros que mais se destacam por serem resistente a vários fatores ambientais é Salmonella. Esta bactéria apresenta estrutura morfológica de bastonete, Gram negativos, são catalase positivas e oxidase negativas, não esporuladas e utilizam compostos químicos orgânicos como principal fontes de energia. Além disso, produzem gás devido ao seu metabolismo oxidativo e fermentativo. Este gênero é um dos principais causadores de enfermidades veiculadas por alimentos, pelo fato de se ter uma adaptação fisiológica que proporciona a multiplicação em valores de pH entre 7,0 e 7,5 e temperaturas entre $35^{\circ} \mathrm{C}$ a $43^{\circ} \mathrm{C}$ e uma atividade hídrica superior a 0,94 (BRASIL, 2001; SOUZA, 2019; HAJDENWURCEL,1998; PIMENTEL,2019).

Os alimentos de origem animal são considerados como principais veículos de transmissão de Salmonella, sendo o leite e queijos os produtos que possuem maior fonte de infecção e/ou intoxicação alimentar, sendo relatado diversos surtos de salmonelose em pessoas 
que consumiram estes produtos, principalmente na forma cru, como ocorre em maior parte do queijo coalho artesanal (BORGES, 2010; SOUZA, 2019).

Importante ressaltar que, embora Salmonella seja uma bactéria com potencial patogênico, a sua inativação ocorre rapidamente em temperatura de pasteurização em alimentos com atividade de água $\geq 0,95$. Entretanto, quando ocorre o tratamento térmico com temperatura inferior, aumenta a termorresistência desta bactéria (BRASIL, 2011).

Outro patógeno veiculados por queijos e outros alimentos, é L. monocytogenes, uma bactéria Gram-positiva, considerada psicotrófica por se multiplicar em temperaturas de refrigeração, e apresentar a capacidade de se desenvolver em ambientes com valores de $\mathrm{pH}$ de até 4,4 (JUNTILLA et al., 1988; ADAMS E MOSS, 1996; BARANCELLI et al., 2011; MATEUS, 2018; DE ABREU CESPI, 2020).

Este patógeno é normalmente veiculado por alimentos crus e/ou, por alimentos em más condições higiênico-sanitárias do ambiente de processamento, e/ ou introduzida no queijo como contaminantes após a pasteurização, se o controlo desse processo não for realizado de maneira adequada, podendo acarretar infecções graves em humanos, com destaque para a listeriose (BELLIO, et al., 2016, CESARO, 2020).

A listeriose é uma doença que pode apresentar quadros clínicos graves, incluindo aborto, morte fetal e parto prematuro, além do desenvolvimento de meningite bacteriana. $\mathrm{O}$ alvo dessa doença normalmente são pessoas com o sistema imunológico comprometido, idosos, crianças e grávidas (EFSA, 2013; GARRIDO, 2009; LIANOU, 2007; BELLIO, et al., 2016; GONÇALVES, 2017; SILVA et al., 2019).

O gênero Staphylococcus pertence à família Micrococcae, e se caracteriza por ser uns cocos Gram positivos, considerado um patógeno oportunista que é facilmente encontrado em pele e mucosa dos seres humanos (QUINN et al., 2007; DE ANDRADE JUNIOR et al., 2019; SANTOS et al., 2007). São microrganismos que possuem a capacidade de multiplicar em temperaturas entre $7^{\circ}$ e $47,8^{\circ} \mathrm{C}$, podendo produzir enterotoxinas em temperaturas entre $10^{\circ} \mathrm{e}$ $46^{\circ} \mathrm{C}$. O seu desenvolvimento pode ocorrer em $\mathrm{pH}$ entre 7 a 7,5; embora possa multiplicar em alimentos que contém pH variando entre 4,2 a 9,3 (SANTANA et al., 2010; SILVA et al., 2017). Os sintomas clínicos ocasionados por $S$. aureus são observadas, em geral, após 4 horas da ingestão da toxina e, dentre os sintomias se destacam: náuseas, vômitos, diarreia e dores abdominais (SILVA et al., 2019).

Diferentes trabalhos na literatura relatam a contaminação por diferentes grupos microbianos em queijo coalho. No trabalho de Araújo, 2017, ao analisar amostras de queijo coalho da região do agreste paraibano, constatou que todas as amostras estavam contaminadas 
com coliformes totais e termotolerantes, inferindo que as BPF não eram realizadas corretamente

(ARAÚJO 2017). Na realização do trabalho De Souza et al., 2020, foram coletadas amostras de queijo coalho na cidade de Soledade na Paraíba, sendo que de sete amostras, cinco continham a presença de Salmonela, demonstrando a ausência da utilização de leite pasteurizado e/ou falha no processo de pasteurização (DE SOUZA et al, 2020). No estudo efetuado por De Oliveira et al, 2019, observaram que de trinta e seis amostras analisadas de queijo de coalho no estado do Ceará, 64,9\% apresentava contagens altas para S. aureus, infringindo os padrões microbiológicos estabelecidos pela legislação vigente, indicando que não houve a realização adequada das condições higiênico-sanitárias (DE OLIVEIRA et al, 2019).

Neste contexto, o diagnóstico da qualidade microbiológica em queijo coalho artesanal produzidos em Nossa Senhora da Glória, maior polo leiteiro do estado de Sergipe, pode contribuir para oferecer aos consumidores um produto mais seguro, com melhor estabilidade microbiológica, e nortear a os produtores deste alimento, sobre possíveis não conformidades nas amostras analisadas com relação a estes parâmetros estabelecidos pela legislação vigente.

\section{METODOLOGIA}

Este projeto foi desenvolvido no Laboratório Multidisciplinar da Universidade Federal de Sergipe, Campus do Sertão, em parceria com a Empresa de Desenvolvimento Agropecuário de Sergipe (EMDAGRO).

\section{Obtenção do queijo coalho artesanal}

Foram coletadas 6 amostras de queijo coalho artesanal de diferentes produtores da região de Nossa Senhora da Glória (SE). Estes diferentes produtores foram escolhidos aleatoriamente em parceria com a EMDAGRO, e que voluntariamente participaram desta pesquisa. Foram incluídos no estudo somente amostras de queijo coalho artesanal sem registro de inspeção (federal, estadual e/ou municipal) e que não apresentaram alterações em suas características sensoriais e macroscópicas.

As amostras coletadas foram armazenadas em caixas isotérmicas com gelo reciclável e termômetro para controle de temperatura, e transportadas ao Laboratório Multidisciplinar da Universidade Federal de Sergipe, campus do Sertão, onde foram submetidas a análises microbiológicas. As amostras dos seis diferentes produtores foram identificadas como $\mathrm{P}_{1}, \mathrm{P}_{2}$, 


\section{Preparo das amostras}

O preparo das amostras, para cada queijo coalho artesanal proveniente de cada produtor, foram divididas, individualmente, em quatro porções aleatoriamente, a fim de se obter uma alíquota de 25 g, mensurada em balança semi analítica (Marte, AS1000C). Em seguida as amostras foram homogeneizadas em um homogeneizador tipo Stomacher (Seward, 400) com $225 \mathrm{ml}$ de solução de salina $0,85 \%$ (Vetec). Todas as análises foram realizadas em duplicata com duas repetições.

\section{Análises microbiológicas}

As análises microbiológicas no queijo coalho artesanal foram determinadas conforme Instrução Normativa $n^{\circ} 62 / 2003$ (BRASIL, 2003) e de acordo com metodologia proposta por Santana et al. com modificações (2008).

\section{Salmonella sp}

Após o preparo, as amostras diluídas foram inicialmente pré-incubadas a $37^{\circ} \mathrm{C}$ por 18 $24 \mathrm{~h}$, e posteriormente, $100 \mu \mathrm{L}$ foram adicionados assepticamente e semeadas em placas Compact Dry Compact SL® (Nissui Pharmaceutical Co., Ltd., Tokyo, Japan) por $41-43^{\circ} \mathrm{C}$ por $24 \mathrm{~h}$. Todas as análises foram realizadas em duplicata e realizadas conforme as instruções do fabricante. Os resultados foram expressos em presença ou ausência de Salmonella sp.em $25 \mathrm{~g}$ da amostra.

\section{Staphylococcus aureus coagulase positiva}

Para cada amostra de queijo coalho artesanal foi utilizado o método spreed plate com diluições apropriadas na superfície de placas contendo Agar Vogel Johnson (Acumedia, Brasil), adicionado da solução de telurito de potássio $1 \%$ (Dinâmica, Brasil) com posterior incubação a $37^{\circ} \mathrm{C}$ por $48 \mathrm{~h}$. Após este período foi verificado a morfologia das colônias, de cores negras, com um halo amarelo em volta. Foram selecionadas as colônias das melhores diluições e transferidas para um tubo com Ágar Triptona de Soja (TSA) (Kasvi, Brasil) inclinado e incubado $37^{\circ} \mathrm{C}$ por $24 \mathrm{~h}$. Em seguida foram realizados testes bioquímicos para 
confirmação como o teste da coagulase, catalase e DNASE e capacidade de utilizar o manitol.

Os resultados foram expressos em Unidade Formadora de Colônia por grama (UFC· grama $\left.{ }^{1}\right)$.

\section{Coliformes termotolerantes}

A quantificação de coliformes termotolerantes foram quantificados pela técnica do Número Mais Provável (NMP) com séries de três tubos. Alíquotas de $1 \mathrm{~mL}$ das diluições das amostras para cada queijo coalho artesanal, foram inoculadas individualmente em tubos com Caldo Lauril Sulfato Triptose (Kasvi, Brasil) com tubos de Durhan invertidos e incubados a $35^{\circ} \mathrm{C}$ por 24 a 48 h. Posteriormente, os tubos que apresentarem resultado positivo (presença de turvação e produção de gás) foram separados para a quantificação de coliformes termotolerantes. Para os coliformes termotolerantes foram utilizados tubos, contendo $10 \mathrm{ml}$ de Caldo Verde Brilhante Bile 2\% (VB) (Acumedia, Brasil), com tubos de Durham invertido, incubados a $37^{\circ} \mathrm{C}$ por 24 a 48 horas, sendo considerados positivos os tubos com turvação do meio e produção de gás. Posteriormente, os tubos que apresentaram resultado positivo foram repicados para tubos contendo Caldo Escherichia coli (EC) (Acumedia, Brasil), e incubados a $44,5^{\circ} \mathrm{C}$ por $48 \mathrm{~h}$. O resultado foi expresso em NMP de coliformes termotorelantes por $\mathrm{g}$ de amostra analisada (NMP. $\left.\mathrm{g}^{-1}\right)$.

\section{Listeria monocytogenes}

Para a quantificação de L. monocytogenes, após o preparo da amostra, $100 \mu \mathrm{L}$ de cada amostra de queijo coalho artesanal, foi adicionada assepticamente e semeadas em placas Compact Dry Compact® (Nissui Pharmaceutical Co. Ltd. Tokyo, Japan) por $35^{\circ} \mathrm{C}$ por 24 horas. Todas as análises foram realizadas em duplicata e realizadas conforme as instruções do fabricante. Os resultados foram expressos em presença ou ausência de L. monocytogenes em $25 \mathrm{~g}$ da amostra.

\section{Análise estatística}

Os resultados obtidos para as amostras de queijo coalho artesanal para cada produtor foram submetidos e analisados com o programa Excel (Windows, 2010) através de análise descritiva e quantitativa e comparados com os critérios microbiológicos estabelecidos pela 

em duplicata com três repetições.

\section{RESULTADOS E DISCUSSÃO}

Os resultados obtidos da quantificaçao de diferentes grupos microbianos nas amostras de queijo coalho artesenal estão evidenciados no Quadro 01.

Quadro 01: Resultados das análises microbiológicas para amostras de queijo de coalho produzidas artesanalmente no município de Nossa Senhora da Glória, Sergipe.

\begin{tabular}{|c|c|c|c|c|c|c|c|}
\hline \multirow{2}{*}{ Microrganismos } & \multicolumn{5}{|c|}{ Produtor } & \multicolumn{1}{|c|}{$\begin{array}{c}\text { I.N } \\
\text { No60/2019 }\end{array}$} \\
\cline { 2 - 7 } & $\mathrm{P} 1$ & $\mathrm{P} 2$ & $\mathrm{P} 3$ & $\mathrm{P} 4$ & $\mathrm{P} 5$ & $\mathrm{P} 6$ & Aus.em \\
Salmonella sp & Aus. & Aus. & Aus. & Presente & Aus. & Presente & $\begin{array}{c}\text { Aug } \\
25 .\end{array}$ \\
\hline $\begin{array}{c}\text { S. aureus } \\
\text { coagulase positiva } \\
\left(\text { UFC. } \mathrm{g}^{-1}\right)\end{array}$ & $3,0 \times 10^{2}$ & $4,2 \times 10^{2}$ & $2,9 \times 10^{1}$ & $1,0 \times 10^{3}$ & $5,8 \times 10^{2}$ & $4,7 \times 10^{3}$ & $10^{2}$ \\
\hline $\begin{array}{c}\text { Coliformes } \\
\text { termotolerantes }\end{array}$ & $2,4 \times 10^{2}$ & $2,2 \times 10^{4}$ & $1,0 \times 10^{3}$ & $1,4 \times 10^{4}$ & $3,1 \times 10^{2}$ & $2,1 \times 10^{4}$ & $10^{2}$ \\
\hline Listeria monocytogenes & Aus. & Aus. & Aus. & Aus. & Aus. & Aus. & $\begin{array}{c}\text { Aus.em } \\
25 \mathrm{~g}\end{array}$ \\
\hline
\end{tabular}

P1 - produtor 1; P2 - produtor 2; P3 - produtor 3; P4 - produtor 4; P5 - produtor 5; P6 - produtor 6; Aus.: ausente; N.P: não se aplica.

Fonte: Própria (2021).

De acordo com resultados obtidos foi possivel verificar que, os produtores de queijo coalho artesenal P4 e P6, tiveram a presença de Salmonella sp, em 25 g de amostra (Qaudro 1), demostrando a não conformidade em 33,33\% das amostras analisadas com os padrões regidos pela IN N 60 de 2019 que estabele, a ausência desse microrganismo em 25 de amostra. Ao analisar amostras de queijos de coalho comercializados no Mercado Central de Aracaju/SE, Santana et al. (2008), observaram que das 60 amostras analisadas, 26,67\% apresentaram a presença de Salmonella sp. Em um percentual menor, Duarte et al. (2005) identificaram a presença desse patógeno em 5,5\% das amostras de queijos coletadas em empreendimentos comerciais no Estado de Pernambuco. A presença de Salmonella sp nas amostras analisadas demostram que o queijo coalho artesanal produzidos pelos produtores P4 e P6 estão impróprios para o consumo, uma vez que este microrganismo apresenta elevado potencial patogênico. No Brasil, entre os anos de 2007 a 2017 foram registrados 7.170 surtos relacionados com doenças veiculadas por alimentos, sendo o leite e os derivados lácteos, como o queijo, responsáveis por 
3.802 dos casos notificados, sendo a bactéria do gênero Salmonella, um dos principais agentes responsáveis por casos mais graves e fatais (BRASIL, 2018; AMARAL, et al., 2020). Importante ressaltar que, se as ferramentas de Boas Práticas de Fabricaçao (BPF) e Boas Práticas de ordenha (BPO) forem negligenciadas durante toda a etapa produtiva da obtençao do leite, poderá haver a contaminação da matéria prima, pois os queijos artesanais são produzidos, majoritariamente, com leite não pasteurizado, favorecendo assim que, bactérias do gênero Salmonella sp, possam aderir e colonizar o trato gastrointestinal dos animais (DUARTE et., 2005, BOMFIM et al., 2020). As amostras que apresentaram a ausência de Salmonella em $25 \mathrm{~g}$, pode ter sido favorecidas pela presença natural de bacterias ácido-lácticas (BALs), uma vez que estudos na literatura reportam que a alta incidência deste grupo microbiano presente no leite cru exercem atividade antagonista para a multiplicação de Salmonella sp. (RIBEIRO, 2019, MELO, et al., 2013, AMAREAL, et al., 2020).

A quantificação de $S$. aureus coagulase positiva demostrou que todas as amostras (100\%) apresentaram resultado insatisfatório em relação a legislação vigente, cujo limite é de $10^{2} \mathrm{UFC} \cdot \mathrm{g}^{-1}$ (Quadro 1). Resultados semelhantes foram reportados por Sousa et al. (2014), que ao analisarem 104 amostras de queijos de coalho de seis estados do Nordeste, observaram contagens elevadas deste microrganismo em $95,16 \%$ das amostras examinadas. No trabalho De Freitas Filho et al. (2020), foi demonstrado que todas as amostras avaliadas apresentaram valores superior ao permitido para $S$. aureus coagulase positiva, oscilando entre $2,5 \times 10^{4}$ a 4,8 $\mathrm{x} 10^{7} \mathrm{UFC} \cdot \mathrm{g}^{-1}$. A quantificaçao de $S$. aureus coagulase positiva superior ao máximo permitido pela legislaçao vigente é uma indicação que, possivelmente, as condiçoes higiênicas-sanitárias com relaçao ao leite utilizado e/ou durante a manipulação e armazenamento dos queijos coalho artesenal analisados foram realizadas de forma insatisfatória, visto que as bactérias do gênero S. aureus fazem parte da microbiota normal de pele, mucosas e narinas, podendo ser veiculada aos alimentos por contato indireto ou direto (AMSON, et al., BOMFIM et al., 2020), com por exemplo, durante a manipulaçao do processamento de queijo coalho artesenal. Salienta-se que, a contagem elevada deste patógeno em queijo coalho artesenal, é um fator preocupante devido a elevada resistência térmica das enterotoxinas produzidas por este microrganismo, podendo propiciar riscos à sáude do consumidor (ALVES et al., 2009; FORSYTHE, 2005), o que torna todas as amostras de queijo coalho artesanal analisadas improprias para o consumo.

De acordo com a IN 60 de 2019 (ANVISA) o limite máximo permitido para coliformes termotolerantes (Escherichia coli) para queijos com umidade igual ou superior a $46 \%$ é de $10^{2}$ $\mathrm{NMP} \cdot \mathrm{g}^{-1}$, sendo possível verificar que, de acordo com os resultados obtidos, todas as amostras apresentaram valores superiores ao permitido pela legislação vigente (Quadro 1). Com base na 
pesquisa realizada por Santos et al. (2018) em queijo coalho comercializado em Maceió/AL, identificou-se que as amostras analisadas para coliformes termotolerantes, estavam em desconformidade segundo a legislação vigente, concluindo como um alimento inapropriado para o consumo, uma vez que esta bácteria pode produzir toxinas no alimento e ocasionar intoxicação alimentar. Além disso, a presença de coliformes termotolerantes em contagens superiores ao recomendando pela legislação, pode ser um forte indicador de contaminação microbiana de material fecal, apontando como causa principal a falta do controle higiênico sanitário adequado, proporcionando risco para a saúde do consumidor (SILVA et al. 2018). Dessa forma, como base nos resultados encontrados (Quadro 01) sugere-se que as Boas Práticas de Fabricação, em especial os aspectos higiênicos-sanitários durante a manipulação do queijo coalho artesanal foram possivelmente negligenciadas, tornado todas amostras pesquisadas inapropriadas para o consumo.

Com relação a quantificação de L. monocytogenes, foi possível verificar que, todas as amostras de queijo coalho artesanal, atenderam aos requisitos da legislação vigente, que preconiza a ausência deste patógeno em $25 \mathrm{~g}$ do produto. Resultado similar foi encontrado no trabalho de Feitosa et al. (2003), com amostras coletadas do Estado do Rio Grande do Norte, onde as 12 amostras de queijo coalho analisadas apresentaram a ausência de L. monocytogenes. Entretanto, é possível verificar na literatura, pesquisas que identificaram a presença de $L$. monocytogenes em queijo coalho artesanal produzidos e comercializados em diferentes estados do Brasil (FEITOSA, et al., 2003; BORGES et al., 2003; GUEDES, 2003, TORRES, 2021). A ausência de L. monocytogenes nas amostras de alimentos, muitas vezes, está relacionada a fatores de estresse celular e à baixa concentração de células do patógeno (BONFIM, 2020).

Neste contexto, embora todas as amostras analisadas de queijo coalho produzidos em Nossa Senhora da Glória, SE, tenham apresentado a ausência de L. monocytogenes, foi possível constar a presença de outros microrganismos patogênicos (Salmonella sp, S. aureus coagulase positiva e coliformes termotolerantes) em quantidades superiores aos limites estabelecidos pela I.N N ${ }^{\circ} 60$ de 2019 (ANVISA), demostrando que, 100\% dos queijos coalho artesanal analisados estavam impróprios para o consumo humano. Enfatiza-se que a implementação das BPF aliada com as Boas Práticas de Ordenha (BPO) e a sensibilização destes produtores em toda a cadeia produtiva do queijo colho artesanal, poderá contribuir para a melhoria dos parâmetros microbiológicos nas amostras analisadas, favorecendo assim para a obtenção de um alimento seguro para o consumo e que atenda aos padrões microbiológicos estabelecidos pela legislação vigente. 
A avalição dos dados microbiológicos demonstraram que, embora constatado a ausência de L monocytogens nas amostras de queijo coalho artesanal, todas as amostras analisadas de queijo coalho artesanal estavam parcialmente ( Salmonella $s p$,) ou totalmente ( . aureus coagulase positiva e coliformes termotolerantes) em não conformidade com a legislação vigente para diferentes microrganismos patogênicos, estando, portanto, inadequadas para o consumo humano, uma vez que, a sua ingestão pode ocasionar infecção e/ou intoxicação alimentar. Importante ressaltar, que a partir dos resultados obtidos, sugere-se que a implementação efetiva de BPF desde a etapa de obtenção do leite até a produção de queijo coalho artesanal, necessita ser aperfeiçoada, para que os parâmetros microbiológicos possam ser melhorados visando desta forma, atender os requisitos da legislação vigente, além de oferecer um alimento microbiologicamente seguro para o consumidor.

\section{REFERÊNCIAS}

Adams, M.R. e Moss, M.O. (1996). Food Microbiology. Pp: 186 - 191. The Royal Society of Chemistry.

ALVES, L. MC., et al. Qualidade Microbiológica do Leite Cru e de Queijo de Coalho Comercializados Informalmente na Cidade de São Luís -MA. Pesquisa em Foco; v. 17, n.2, p. 01-13, 2009.

AMARAL, José Wilker, et al. Avaliação da qualidade de queijos de produção informal. Segur. Aliment. Nutr., Campinas, v. 27, p. 1-6. e020016, 2020.

AMSON, G. V. et al. Levantamento de dados epidemiológicos relativos à ocorrências/ surtos de doenças transmitidas por alimentos (DTAs) no estado do Paraná Brasil, no período de 1978 a 2000. Ciênc. agrotec, Lavras, vol.30, n.6, p.1139-1145,2006.

ARAUJO, Rayane Maria da Silva. Pesquisa de coliformes totais e coliformes termotolerantes em queijos tipo coalho produzidos com leite cru na região do agreste paraibano. Trabalho de Conclusao de Curso, Universidade Federal da Paraíba, 2017, 25p.

BARANCELLI et al. Listeria monocytogenes: Ocorrência em produtos lácteos e suas implicações em saúde pública. São Paulo: Arquivo do Instituto Biológico, vol. 78, no. 1, 2011.

BARBOZA, Giovana Rueda et al. Perfil de resistência e virulência de Escherichia coli diarreiogênicas isoladas de plantas de processamento de queijo tipo minas frescal. 2018.

BELliO, A., Astegiano, S., Traversa, A., Bianchi, D. M., Gallina, S., Vitale, N., Zuccon, F. \& Decastelli, L. (2016). Behaviour of Listeria monocytogenes and Staphylococcus aureus in 
sliced, vacuum-packaged raw milk cheese stored at two different temperatures and time periods. International Dairy Journal, 57, 15-19. Doi:10.1016/j.idairyj.2016.02.003.

BOMFIM, Adriele Pinheiro, et al. Qualidade microbiológica e caracterização da resistência antimicrobiana de bactérias isoladas de queijos Coalho comercializados em Vitória da Conquista-Bahia. Segur. Aliment. Nutr., Campinas, v. 27, p. 1-10. e020015, 2020.

BORGES,M.F. et al. Micro-organismos Patogênicos e Indicadores em Queijo de Coalho Produzido no Estado do Ceará, Brasil.Boletim do Centro de Pesquisa de Processamento de Alimentos. Curitiba, v. 21, n. 1, jan./jun., 2003.

BORGES, M. de F.; ANDRADE, APC DE; MACHADO, Terezinha Feitosa. Salmonelose associada ao consumo de leite e produtos lácteos. Embrapa Agroindústria TropicalDocumentos (INFOTECA-E), 2010.

BRASIL. Ministério da Agricultura e do abastecimento. Instrução Normativa $N^{\circ} 30$, de 26. Jun. 2001. Regulamento Técnico de Identidade e Qualidade do Queijo Coalho. Brasília: Ministério da Agricultura e do Abastecimento, 2001.

BRASIL. Ministério da Agricultura, Pecuária e Abastecimento. Departamento de Inspeção de Produtos de Origem Animal. Instrução Normativa $\mathbf{N}^{\circ} .62$, de 26 de agosto de 2003. Oficializa os Métodos Analíticos Oficiais para Análises Microbiológicas para Controle de Produtos de Origem Animal e Água. Diário Oficial da República Federativa do Brasil, p. 14, 18 set. 2003. Seção I.

BRASIL. Ministério Da Saúde. Secretaria De Vigilância Em Saúde. Manual técnico de diagnóstico laboratorial de Salmonella spp.: diagnóstico laboratorial do gênero Salmonella. 2011.

BRASIL. Ministério da Agricultura e do abastecimento. Instrução Normativa No57, de 15 de Dez de 2011. Brasília: Ministério da Agricultura e do Abastecimento, 2011.

BRASIL. Surtos de Doenças Transmitidas por Alimentos no Brasil. Ministério da Saúde [documento eletrônico] 2018 [citado em 2019 out 22]; Disponível em: https://portalarquivos2.saude.gov.br/images/pdf/2018/ janeiro/17/Apresentacao-Surtos-DTA2018.pdf.

BRASIL, Instituto Brasileiro de Geografia e Estatística, Brasil 2015/2019. Disponível em: https://sidra.ibge.gov.br/home/ipp/brasil . Acesso em: 03/05/2021.

BRASIL. LEI No 8523, de 29. Abr. 2019. Assembleia Legislativa do Estado de Sergipe, Aracaju, SE. Disponível em: https://www.normasbrasil.com.br/norma/lei-8523-2019se 377115.html. Acesso em: 27 Abr. 2021.

BRASIL. Ministério da Agricultura e do abastecimento. Instrução Normativa $\mathrm{N}^{\circ}$ 60, de 23 de deze de 2019. Brasília: Ministério da Agricultura e do Abastecimento, 2019.

CARDOSO ALSP, Tessari ENC, Castro AGM, Kanashiro AMI, Gama NMSQ. Pesquisa de coliformes totais e coliformes fecais analisados em ovos comerciais no laboratório de patologia avícola de descalvado. Arq. Inst. Biol, 68(1):19-22, 2001. 
CRUZ, A.G.; BURITI, F.C.A.; SOUZA, C.H.B. et al. Probiotic cheese: health benefits, technological and stability aspects. Trends in Food Science \& Technology, v.20, p.344-354, 2009.

CAVALCANTE, José Fernando Mourão et al. Processamento do queijo coalho regional empregando leite pasteurizado e cultura lática endógena. Food Science and Technology, v. 27, n. 1, p. 205-214, 2007.

CAVALCANTE, J. F. M. Sistema de Apoio à decisão na produção de leite e queijo coalho com segurança alimentar. 2005. 158f. Tese (Doutorado em Ciência e Tecnologia dos Alimentos). Universidade Federal de Viçosa. MG. 2005.

CESARO, Elisângela de. Elaboração de queijo de leite cru maturado com cobertura de erva-mate (Ilex paraguariensis) (Mestrado), Universidade Estadual de Maringá, 2020, 60p.

CUNHA MA. Métodos de detecção de microorganismos indicadores. Saúde \& Ambiente em Revista, 1(1):09-13, 2006.

DE ABREU CESPI, Beatriz; OLIVEIRA, Raquel; KUNIGK, Cynthia Jurkiewicz. Modelagem do comportamento de Listeria Innocua durante a produção de queijo artesanal.Disponivel em https://maua.br/files/122020/modelagem-do-comportamento-listeria-innocua-duranteproducao-queijo-artesanal-151417.pdf. Acesso em 10 de abril, 2021.

DE ANDRADE JÚNIOR, Francisco Patricio et al. Fatores que propiciam o desenvolvimento de Staphylococcus aureus em alimentos e riscos atrelados a contaminação: uma breve revisão. Revista de Ciências Médicas e Biológicas, v. 18, n. 1, p. 89-93, 2019.

DE FREITAS FILHO, João Rufino et al. Avaliação dos parâmetros físico-químicos e microbiológicos de queijo coalho comercializado em feiras livres. Almanaque Multidisciplinar de Pesquisa, v. 7, n. 2, 2020.

DE OLIVEIRA, Francisco Irineu Pereira et al. Ocorrência de Staphylococcus aureus em queijos tipo coalho. Cadernos ESP-Revista Científica da Escola de Saúde Pública do Ceará, v. 13, n. 2, p. 82-93, 2019.

DE SOUSA, Maria Lizieda Fabricio et al. Avaliação microbiológica do queijo artesanal produzido e comercializado em uma cidade do interior da Paraíba. Research, Society and Development, v. 9, n. 8, 2020.

DRUMOND, Sheila Neves; SANTIAGO, Aníbal da Fonseca; MOREIRA, Mariana; LANNA, Maria Célia da Silva; ROESER, Hubert Mathias Peter. Identificação molecular de Escherichia coli diarreiogênica na Bacia Hidrográfica do Rio Xopotó na região do Alto Rio Doce. Engenharia Sanitaria e Ambiental, [S.L.], v. 23, n. 3, p. 579-590, jun. 2018. FapUNIFESP (SciELO).

DUARTE, D.A.M.; SCHUCH, D.M.T.; SANTOS, S.B.; RIBEIRO, A.R.; VASCONCELOS, A.M.M.; SILVA, J.V.D.; MOTA, R.A. Pesquisa de Listeria monocytogenes e microrganismos indicadores higiênicos-sanititários em queijo de coalho produzido e comercializado no Estado de Pernambuco. Arquivos do Instituto Biológico, São Paulo, v.72, n.3, p.297-302, 2005. 
Texas, 2004. 182f. Dissertação (Mestrado em Tecnologia) - Pós-graduação da Texas Tech University, Texas Tech University, Texas, 2004.

ECKERT, Raquel Goreti; WEBBER, Miriam. Controle de qualidade microbiológico de queijos maturados comercializado na feira do pequeno produtor da cidade de Cascavel-PR. Hig. aliment, v. 30, n. 252/253, p. 80-85, 2016.

European Food Safety Authority: Analysis of the baseline survey on the prevalence of Listeria monocytogenes in certain ready-to-eat foods in the EU, 2010-2011. Part A: Listeria monocytogenes prevalence estimates. EFSA J, 11:3241, 2013.

FEITOSA, Terezinha; BORGES, Maria de Fátima; NASSU, Renata Tieko; AZEVEDO, Érika Hardy Franco de; MUNIZ, Celli Rodrigues. Pesquisa de Salmonella sp., Listeria sp. e microrganismos indicadores higiênico-sanitários em queijos produzidos no estado do Rio Grande do Norte. Ciência e Tecnologia de Alimentos, [S.L.], v. 23, p. 162-165, dez. 2003.

FORSYTHE, S. J. Microbiologia da segurança alimentar.Ed. I. Porto Alegre, Brasil: Artmed; 2005.

FRAGA, E. E. A.; OLIVEIRA, C. R.; CRUZ, C. A. B; SANTOS, N. J. B.; VASCONCELOS, C. R.; SANTANA, J. R.. Analysis of the feasibility of geographical indication of the craft production of cook cheese in sergipan area. Revista Gestão Inovação e Tecnologias, [S.L.], v. 9, n. 2, p. 4863-4875, 2019.

FREITAS, Monica Paul. Avaliação microbiológica de queijos artesanais produzidos na cidade de Taió, Santa Catarina. Saúde e meio ambiente: Revista interdisciplinar, v. 4, n. 2, p. 103$114,2015$.

FROZI, Jesieli Braz. Multiplicação e sobrevivência de escherichia coli produtora de toxina shiga (STEC) do sorotipo O157: H7 durante o processamento e armazenamento de queijo minas frescal. (Mestrado). Universidade Federal Fluminense, 2017, 76p.

GARRIDO V, Vitas AI, García I: Survey of Listeria monocytogenes in ready-to-eat products: prevalence by brands and retail establishments for exposure assessment of listeriosis in Northern Spain. Food Control, 20:986- 991, 2009.

GONÇALVES, Magda et al. Presença de Listeria monocytogenes em Queijos de Pasta Mole da Região a Sul do Tejo. Portuguese Journal of Public Health, v. 35, n. 1, p. 37-43, 2017.

HAJDENWURCEL, J. R. Atlas de microbiologia de alimentos, v. 1. São Paulo: Fonte Comunicação e Editora, 1998. 66p.

GUEDES, V.T.M. Ocorrência de Listeria monocytogenes em queijos artesanais comercializados na cidade de Manaus -AM. [Dissertação].Manaus:Universidade Federal do Amazonas -UFAM, 2003.

IBGE. Censo Agropecuário Nossa Senhora Da Glória/ SE. 2017. Disponível em: https://cidades.ibge.gov.br/brasil/se/nossa-senhora-da-gloria/pesquisa/24/0. Acesso em: 07 mar. 2021. 
JESUS, Jusimaria de; MOTA, Flávia Nunes; TAVARES, Mayara dos Santos; SILVA JUNIOR, José Espínola da; REIS, Igor Adriano de Oliveira. Avaliação Microbiológica Do Queijo Requeijão Comercializado No Município De Nossa Senhora Da Glória-Se. Interfaces Científicas - Saúde e Ambiente, [S.L.], v. 7, n. 1, p. 17-26, 17, 2018.

JUNTTILA et al. Minimum growth temperatures of Listeria monocytogenes and nonhaemolytic Listeria. Reino Unido: Journal of Applied Bacteriology, vol. 65, issue 4, 1998.

KASK, S.; ADAMBERG, K.; ORLOWSKI, A. et al.; Physiological properties of Lactobacillus paracasei, L. danicus and L. curvatus strains isolated from Estonian semi-hard cheese. Food Research International, v.36, p.1037-1046, 2003

LIANOU A, Sofos JN: A review of the incidence and transmission of Listeria monocytogenes in ready-to-eat products in retail and food service environments. J Food Protect, 9:2004-2217, 2007.

MATEUS, Teresa Letra et al. Listeria e Listeria monocytogenes em alimentos. TecnoAlimentar, n. 12, p. 56-59, 2018.

MELO, F. D, et al. Avaliação da Inocuidade e Qualidade Microbiológica do Queijo Artesanal Serrano e Sua Relação com as Variáveis Físico Químicas e o Período de Maturação. Acta Scientiae Veterinariae, 41: 1-7, 2013.

MELO, Daniela dos Santos. Avaliação da qualidade de leite crú produzido no alto sertão sergipano frente aos parâmetros preconizados pela legislação. 2020. 50 f. TCC (Graduação) - Curso de Agroindústria, Universidade Federal de Sergipe, Nossa Senhora da Glória - SE, 2020.

MENEZES, S. S.M. CAVALCANTE, J. F. M. Queijo coalho artesanal no estado de Sergipe, p.163-191, in: Queijo Coalho artesanal do Nordeste do Brasil, 248p, 2017.

MENEZES, S. S. M. CRUZ, F. T. MENASCHE, R. Queijo de Coalho e Queijo Artesanal Serrano: Identidades de Produtores e de Consumidores Associadas a Atributos de Qualidade. n. Anais. VIII Congresso Latino-americano de Sociologia Rural. Porto de Galinhas (PE), 2010, p.18.

MENEZES, S. S. M. Fabriquetas de queijo e desenvolvimento territorial do sistema agroalimentar de Sergipe. In: VI Congresso Brasileiro De Geógrafos, Goiânia. Caderno de resumos, 2004. p. 488 e 489.

PIMENTEL, Erika Tavares et al. Qualidade de queijo coalho comercializado em Manaus, AM. ( mestrado). Universidade Federal do Amazonas, 2019, 51p.

QUINN, P. J. et al. Medicina veterinária e doenças infecciosas. Porto Alegre: ARTMED, 2007.

REID, T. M. S. A case of study of cheese associated E. coli O157 outbreaks in Scotland. In: DUFFY, G.; GARVEY, P. Verotoxigenic Escherichia coli. Connecticut: Food \& Nutrition Press, 472 p. cap. 10, p. 201-212, 2001.

RIBEIRO, J.L. Potencial Tecnológico, Probiótico e Antagonista da Microbiota Lática de 
Leite de Búfalas [tese]. Brasília: Universidade de Brasília/ Faculdade de Agronomia e

Veterinária; 2019. 109 p.

SALES, Willian Barbosa et al. Ocorrência de Coliformes Totais e Termotolerantes em pastéis fritos vendidos em bares no centro de Curitiba-PR. Demetra: alimentação, nutrição \& saúde, v. 10, n. 1, p. 77-85, 2015.

SANTANA, R. F.; SANTOS, D. M.; MARTINEZ, A. C. C.; LIMA, Á. S. (2008) Qualidade microbiológica de queijo-coalho comercializado em Aracaju, SE. Arq Bras Med Vet Zootec., v. 60 , n. 6 , p. $1517-1522$.

SANTANA, R. F. et al. Qualidade microbiológica de queijo-coalho comercializado em Aracaju, SE. Arquivo Brasileiro de Medicina Veterinária e Zootecnia, v. 60, n. 6, p. 1517 $1522,2008$.

SANTANA, E.H.W.; BELOTI, V.; ARAGONALEGRO, L.C.; MENDONÇA, M.B.O.C. Estafilococos em alimentos. Arquivos do Instituto Biológico.v. 77, n.3, p.545-554, 2010.

SANTOS, André Luis dos et al. Staphylococcus aureus: visitando uma cepa de importância hospitalar. Jornal Brasileiro de Patologia e Medicina Laboratorial, v. 43, n. 6, p. 413-423, 2007.

SANTOS, Joanna Silva et al. Diagnóstico das condições de processamento de produtos artesanais derivados do leite no Estado de Sergipe. Rev. Inst. Latic. "Cândido Tostes", $\mathrm{n}^{\mathrm{o}}$ 363, 63: 17-25, 2008.

SANTOS, N. S. et al. Avaliação microbiológica em queijo coalho, comercializado em Maceió, AL . Braz. J. of Develop, v. 5, n. 7, p.9271-9281, 2018.

SANTOS, Thaís Costa. Diagnóstico do ambiente de ordenha em propriedades rurais do alto sertão sergipano. 2020. 52 f. TCC (Graduação) - Curso de Agroindústria, Universidade Federal de Sergipe, Nossa Senhora da Glória - Se, 2020.

SERGIPE, Assembleia Legislativa do Estado de Sergipe, LEI N . 8.523 DE 29 DE ABRIL DE 2019. Dispõe normas sobre a produção e a comercialização dos queijos artesanais (tradicional e inovação), 2019.

SILVA, Juliana Fonseca Moreira; FEITOSA, Amanda Campos; RODRIGUES, Rosimeire Mendes. Staphylococcus aureus em alimentos. DESAFIOS-Revista Interdisciplinar Da Universidade Federal Do Tocantins, v. 4, n. 4, p. 15-31, 2017.

SILVA, G. L. et al. Aspectos microbiológicos de queijos coalhos comercializados em feiras livres do município de Petrolina-PE. R. bras. Tecnol. Agroindustr, Ponta Grossa, v. 12, n. 1, p. 2613-2626, 2018.

SILVA, Gabriela Santana da; CASTRO, Renata Cruz de; COSTA JÚNIOR, Carlos Eduardo de Oliveira. Ocorrências de micro-organismos patogênicos presentes em surtos de doenças transmitidas por alimentos (DTAs) no estado de Pernambuco no período de 2012 a 2014. 
SOUSA, Andréa Zilá Barroso de et al. Aspectos físico-químicos e microbiológicos do queijo tipo coalho comercializado em estados do nordeste do Brasil. Arquivos do Instituto Biológico, v. 81, n. 1, p. 30-35, 2014.

SOUZA, Débora Lívia Marcolino de et al. Avaliação físico-química do queijo coalho artesanal e industrial fabricado em Salgueiro-PE. In: $1^{\circ}$ Congresso Internacional Das Ciências Agrárias, 01., 2016, Salgueiro - Pe. Anais [...]. Salgueiro - Pe: Anais, 2016. p. 1-6.

SOUZA, Mailza Gonçalves de. Caracterização físico-química e microbiológica do queijo coalho, com e sem registros, comercializado durante o período seco e chuvoso na cidade de Serra Talhada - PE. 2019. 46 f. TCC (Graduação) - Curso de Zootecnia, Universidade Federal Rural de Pernambuco, Serra Talhada - Pe, 2019.

SVS - Secretaria De Vigilância Em Saúde. Surtos de Doenças Transmitidas por Alimentos no Brasil, $2018 . \quad$ Disponível em: https://portalarquivos2.saude.gov.br/images/pdf/2018/janeiro/17/Apresentacao-Surtos-DTA2018.pdf . Acessado em 23.01.2021.

TEIXEIRA, K.M; OLIVEIRA, A.A. Uso de boas práticas nos sistemas de produção d leite, em Sergipe. ISSN 1678-1953, Documento N 182, Empresa Brasileira de Pesquisa Agropecuária Embrapa Tabuleiros Costeiros Ministério da Agricultura, Pecuária e Abastecimento, dezembro de 2013.

TORRES, A.R.S. et al. Ocorrência de Listeria monocytogenes em queijos coalho e manteiga comercializados fatiados no comércio varejista de Arapiraca - AL. Research, Society and Development, v. 10, n. 1, e26410111775, 2021.

VIEIRA, Lício Valério Lima; SOARES, Roberta Nascimento Gonçalves. Produtos associados ao turismo com potencial de indicação geográfica na Rota do Sertão de Sergipe. Turismo em Foco Volume 2, p. 2019. 